\title{
Survival in Anaplastic Thyroid Carcinoma: A Population-Based Analysis 1973-2015
}

\author{
M. Gore
}

\section{ABSTRACT}

\begin{abstract}
Anaplastic thyroid carcinoma (ATC) is the least common and most aggressive of the thyroid cancers. ATC typically represents less than 5\% of all thyroid cancer diagnoses but represents up to $50 \%$ of thyroid cancer deaths. The rarity of ATC lends itself to study by population-based studies. Methods. The most recent 1973-2015 Surveillance, Epidemiology, and End Results (SEER) database was queried for patients with a diagnosis of ATC. Data on demographics, overall survival, surgical treatment, and staging were extracted. Kaplan-Meier and log-rank analysis was used to evaluate for univariate survival differences, and logistic regression analysis was used to conduct a multivariate analysis. Results. A total of 1642 patients were identified (1021 female, 621 male). The largest age group was $75-79$ years old, with the majority of patients older than 60. Univariate analysis showed that age at diagnosis, AJCC overall stage, T, N, and M stage, and surgical treatment vs. nonsurgical treatment significantly affected overall survival. Conclusions. Anaplastic thyroid carcinoma is a rare and aggressive malignancy. It has a relatively dismal prognosis, but younger age, surgical treatment, and lower stage improve overall survival outcomes.
\end{abstract}

Keywords: Anaplastic thyroid carcinoma, head and neck cancer, kaplanmeier, seer, survival.

\section{INTRODUCTION}

Anaplastic thyroid carcinoma (ATC) is the least common of the four main types of thyroid cancer (papillary, follicular, medullary, and anaplastic) [1]. In contrast to the more differentiated thyroid carcinomas (papillary and follicular carcinoma), ATC behaves in a very clinically aggressive manner, and while ATC only represents less than $5 \%$ of the total thyroid cancer incidence, ATC accounts for approximately $15 \%$ to $50 \%$ of total thyroid cancer deaths. Historically the reported five-year actuarial survival in patients with ATC is typically less than $10 \%$. From a demographic standpoint, ATC is typically seen in older patients ( $>60$ years of age). Consistent with its aggressive phenotype, ATC is often noted to invade local anterior/central neck structures such as the recurrent laryngeal nerve and tracheal cartilages, and ATC has often metastasized to regional lymph nodes or distant sites at the time of diagnosis. Given the aggressive invasion into local structures and the notably high rate of lymphatic and distant metastatic spread, ATC can represent a significant treatment challenge for surgeons, radiation oncologists, and medical oncologists, and a significant proportion of ATC patients have unresectable disease at the time of diagnosis of their malignancy. In the subset of patients with resectable disease at the time of diagnosis surgery combined with post-operative radiation + chemotherapy may be beneficial [1]. The relative rarity of ATC vs the more common well-differentiated thyroid malignancies makes population-based studies a useful tool for examining relevant survival factors in patients with ATC [2]. The present study utilizes the population-based Surveillance, Epidemiology, and End Results (SEER) database and a univariate and multivariate analysis to elucidate factors affecting actuarial survival in patients diagnosed with and treated for ATC in the United States.

\section{METHODS}

The Surveillance, Epidemiology, and End Results (SEER) database is a population-based database maintained by the National Cancer Institute (NCI). The SEER database is updated regularly by the National Cancer Institute and the data is available to researchers who request database access. The database collects and compiles data on cancer cases from various health care centers and hospitals throughout the United States. The most recent SEER database contains available patient data from 1973-2015 and is available at https://seer. cancer.gov/. The most recent SEER data base with patient data up to 2015 was searched for anaplastic thyroid carcinoma in any patient age. The relevant deidentified patient data was compiled and extracted using the dedicated database search program SEER*Stat version 8.3.4 (National Cancer Institute, Bethesda, Maryland) and the data was subsequently exported into Microsoft Excel 2016 (Microsoft Corporation, Redmond, Washington) for analysis. The commercially available statistics program XLstat Biomed (Addinsoft, New York City, NY/Paris, France) was used to determine actuarial Kaplan-Meier overall survival. 
The log-rank method was used to examine univariate factors affecting overall survival. For multivariate survival factors logistical regression analysis was utilized. Statistical significance was set at 0.05. Available 1973-2015 SEER collected and analyzed included overall patient survival, patient age at the time of diagnosis of ATC, patient sex, patient race as reported in the SEER database, AJCC (American Joint Committee on Cancer) stage, tumor (T) stage, nodal (N) stage, and distant metastasis (M) stage. The use of or omission of surgical treatment was also examined in this study. When patient data on a given variable was not available in the SEER database it was noted as unknown.

\section{RESULTS}

A total of 1642 patients with ATC were identified in the 1973-2015 SEER database and were available for analysis in this study. Table I compiles the available demographic data from the present ATC cohort. A ratio of 1.6: 1 (1021 female: 621 male) was found for female vs. male patients in this study. The most common ethnic group in the patient cohort was white/Caucasian with $1322 / 1642$ or $80.5 \%$ of the total patients. Most patients in the SEER ATC cohort were older than 60 years of age. The 70 to 74 and $85+$ age groups represented the largest patient age groups in the study. The AJCC stage data from the group is noted in Table II. As seen in Table II Stage IVC was the largest overall stage subgroup with $458 / 1642$ patients or $27.9 \%$ of the total patient group. For patients for whom tumor $(\mathrm{T})$ stage data was recorded in the SEER database, most were stage T4b (742/1642, or $45.2 \%$ of the cohort). When $\mathrm{N}$ stage was examined, a total of $516 / 1642$ patients, or $31.4 \%$, were $\mathrm{N}+$ (nodal metastasis + ; N1NOS 89/1642, 5.4\%; N1a 102/1642, 6.2\%; N1b 325/1642, $19.8 \%$ ). A relatively high proportion of ATC patients in the SEER database had distant metastases at the time of diagnosis; of patients for whom distant metastasis (M) status was known, 499 of 1642 patients were M0 (30.4\%), while 458 of 1642 patients were M1 (27.9\%).

Fig. 1 demonstrates the Kaplan-Meier overall survival for the cohort. Five-, 10-, and 20- year overall survival was $6.6 \%$, $5.2 \%$, and $2.7 \%$, respectively. Fig. 2 illustrates the overall survival for patients with ATC by patient age at diagnosis. Five-year overall survival was significantly greater for patients less than age 45 at diagnosis than for patients of aged 45 or greater at diagnosis $(p<0.0001)$. Fig. 3 shows the overall survival for ATC patients by patient race. There was no significant difference in overall survival at five years for different racial groups $(p=0.2)$. Fig. 4 demonstrates the Kaplan-Meier actuarial overall survival for patients by patient sex. The study found no significant difference in overall survival at five years for male vs. female patients $(p=0.1)$. Fig. 5 illustrates the Kaplan-Meier actuarial overall survival for patients by the use of or omission of surgical treatment. Overall survival at five years for patients on whom surgery was performed or patients who refused surgery was greater than all other groups not treated with surgery $(p<0.0001)$. Fig. 6 demonstrates the overall survival for patients with ATC according to tumor (T) stage. Overall survival at five years was significantly greater for stage $\mathrm{T} 4 \mathrm{a}$ (tumor confined to the thyroid gland) than for patients with $\mathrm{T} 4 \mathrm{~b}$ disease (extrathyroidal spread present), T4NOS (not otherwise specified), or unknown $\mathrm{T}$ stage patients $(\mathrm{p}<0.0001)$. Fig. 7 notes the actuarial overall survival for ATC patients according to nodal $(\mathrm{N})$ stage. Overall survival at five years was higher for patients with N0 and N1a disease vs. patients with N1b, N1NOS, or NX disease $(\mathrm{p}<0.0001)$. Fig. 8 shows the overall survival for patients by metastasis (M) stage. Overall survival in the ATC cohort at five years was noted to be greater for M0 ATC patients than for M1 ATC patients $(p<0.0001)$. Fig. 9 illustrates the overall survival for ATC patients by AJCC stage. Five year overall actuarial survival was greater for stage IVA patients than stage IVB, IVNOS, or IVC ATC patients $(\mathrm{p}<0.0001)$. Logistic regression analysis was used to perform a multivariate analysis of overall survival. The multivariate analysis data for ATC patients in the cohort is noted in Table III. Multivariate analysis demonstrated that age at diagnosis $(\mathrm{p}<0.0001)$, overall AJCC stage $(p<0.0001)$, T stage $(p<0.0001), N$ stage $(p<0.0001), M$ stage $(p<0.0001)$, and treatment type $(p<0.0001)$ remained significant predictors of overall survival. Race and sex were nonsignificant predictors of survival on multivariate analysis.

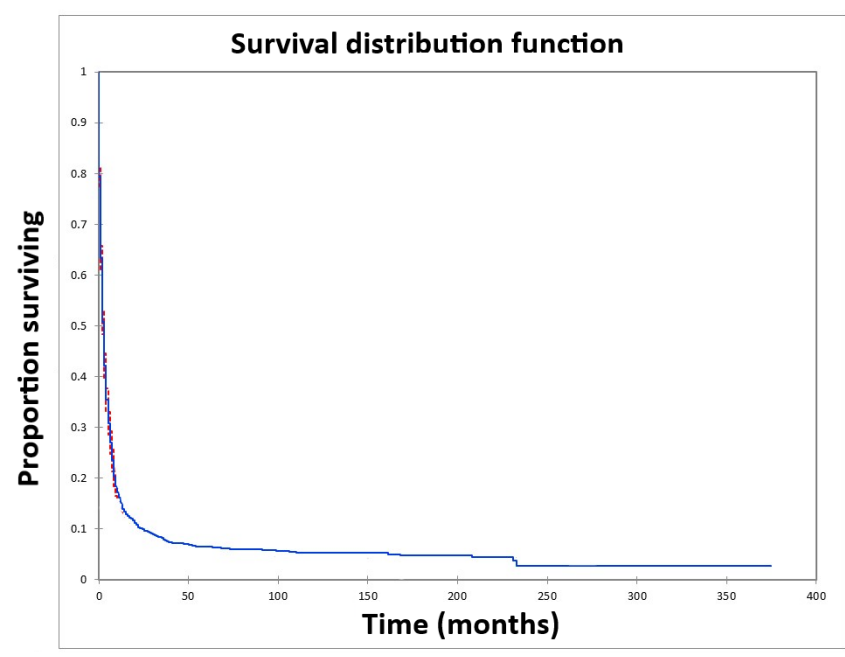

Fig. 1. Kaplan-Meier overall survival for entire cohort.

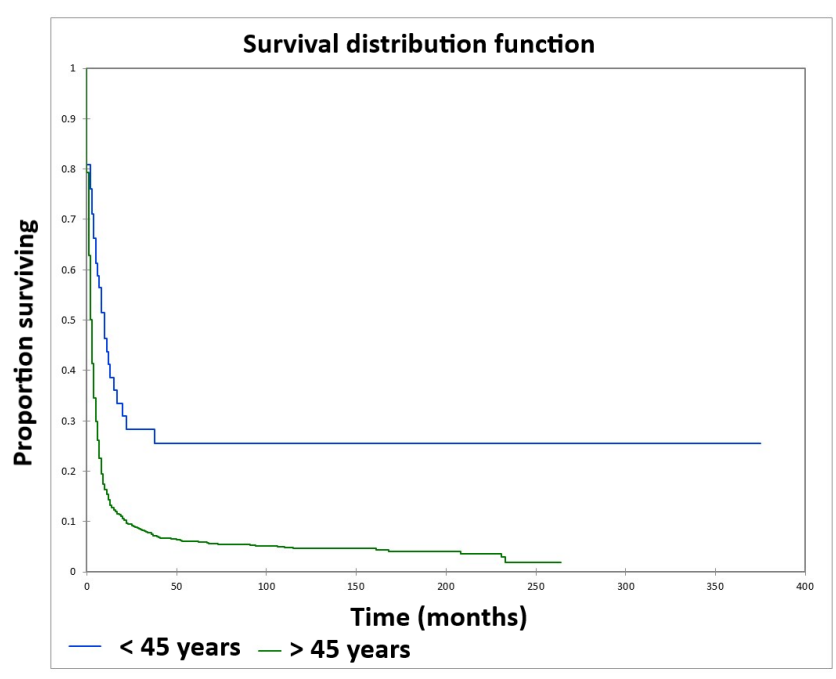

Fig. 2. Kaplan-Meier overall survival by age. 


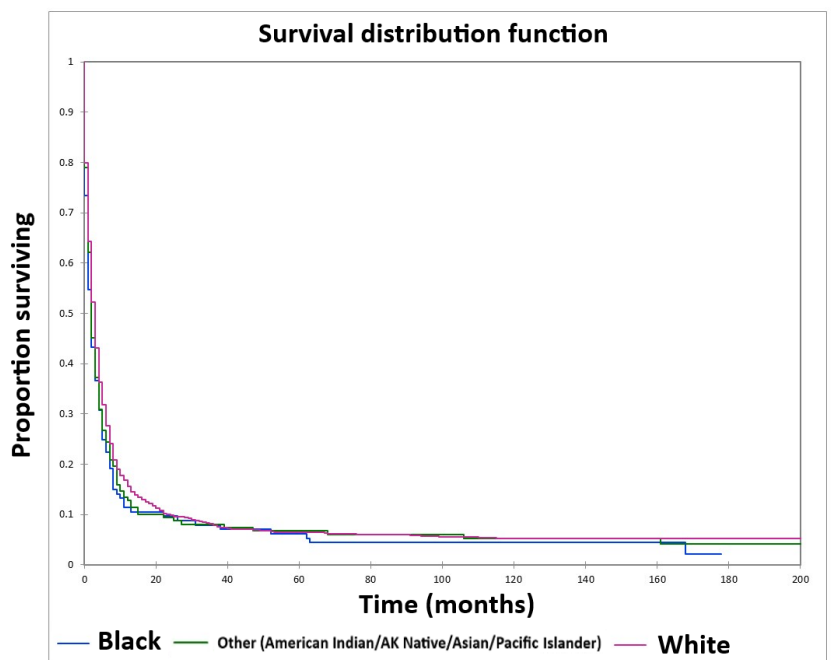

Fig. 3. Kaplan-Meier overall survival by race.

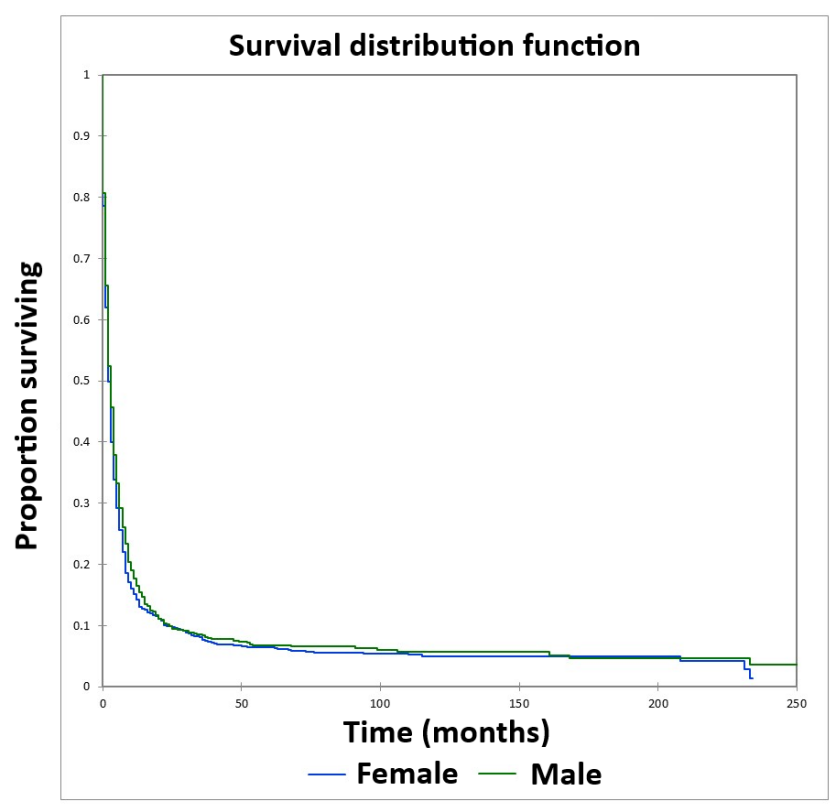

Fig. 4. Kaplan-Meier overall survival by sex.

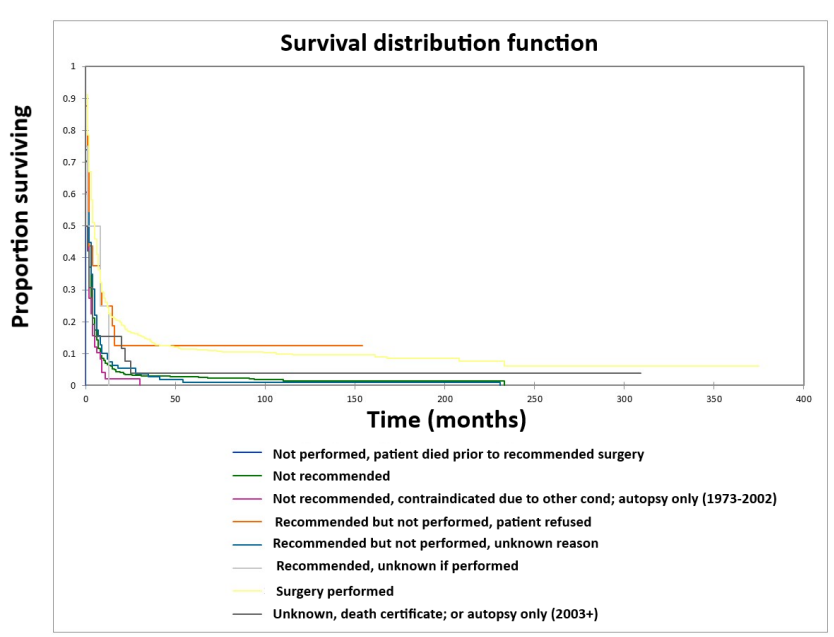

Fig. 5. Kaplan-Meier overall survival by treatment type.

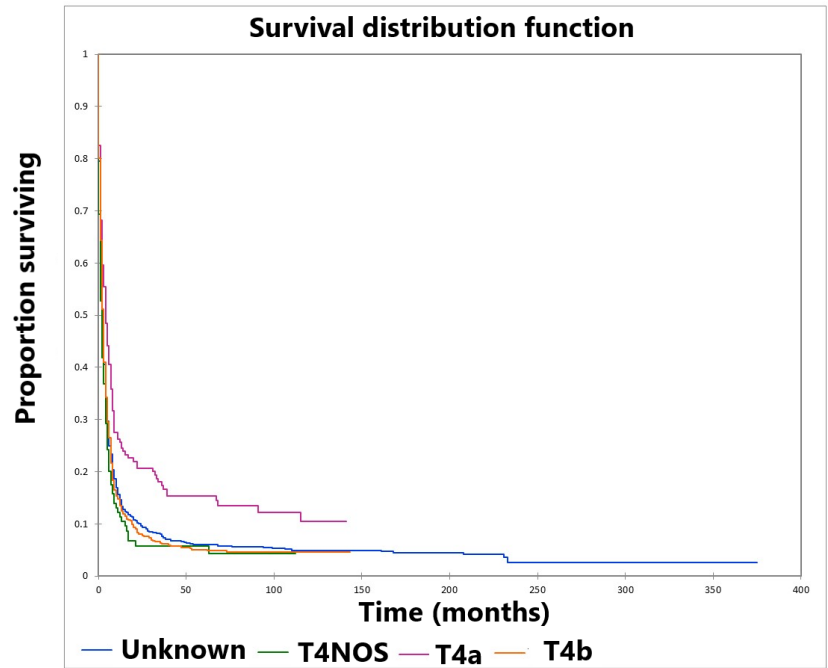

Figure 6 Kaplan-Meier overall survival by T stage.

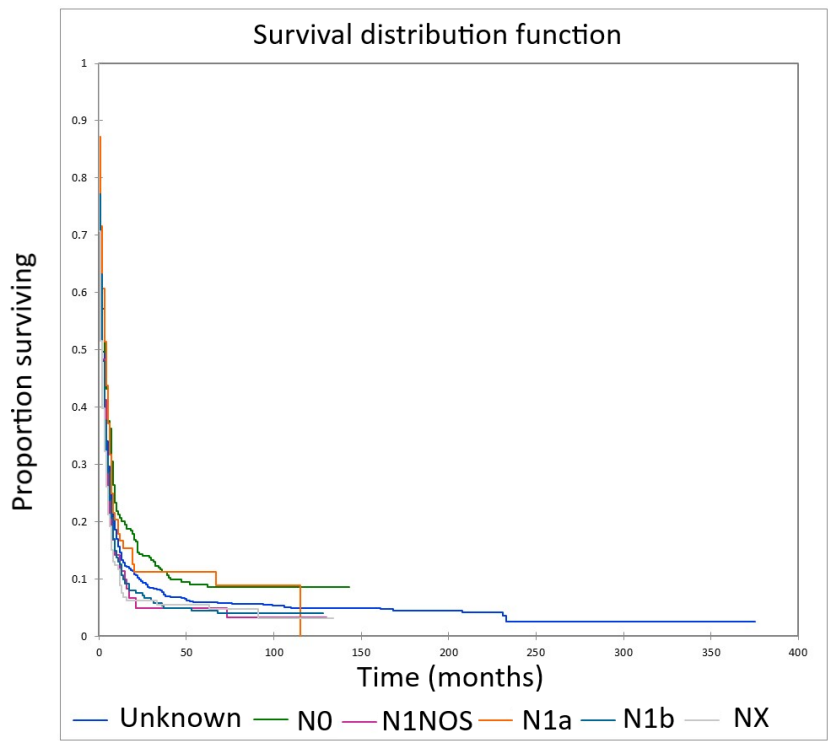

Fig. 7. Kaplan-Meier overall survival by $\mathrm{N}$ stage.

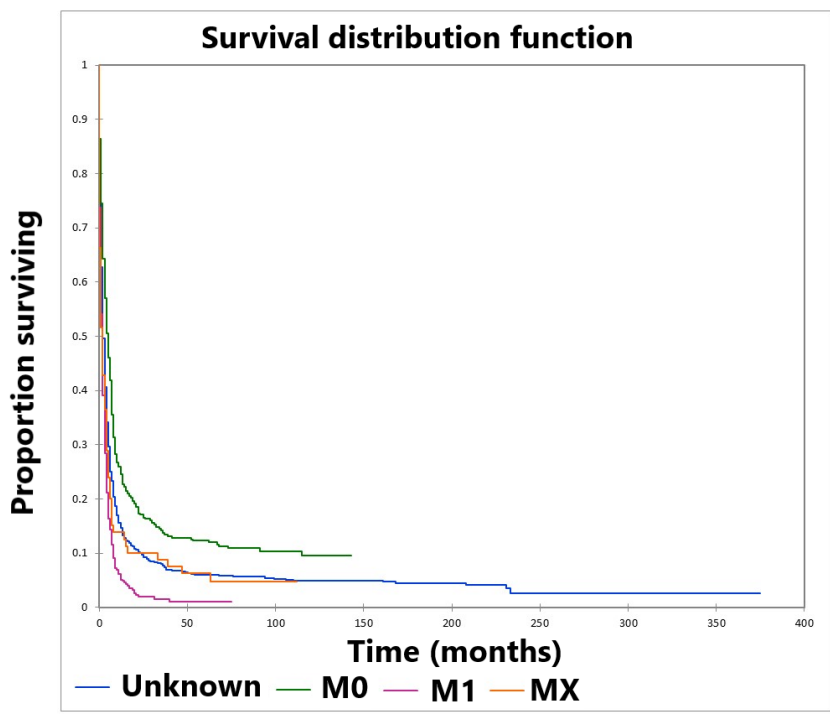

Fig. 8. Kaplan-Meier overall survival by $M$ stage. 


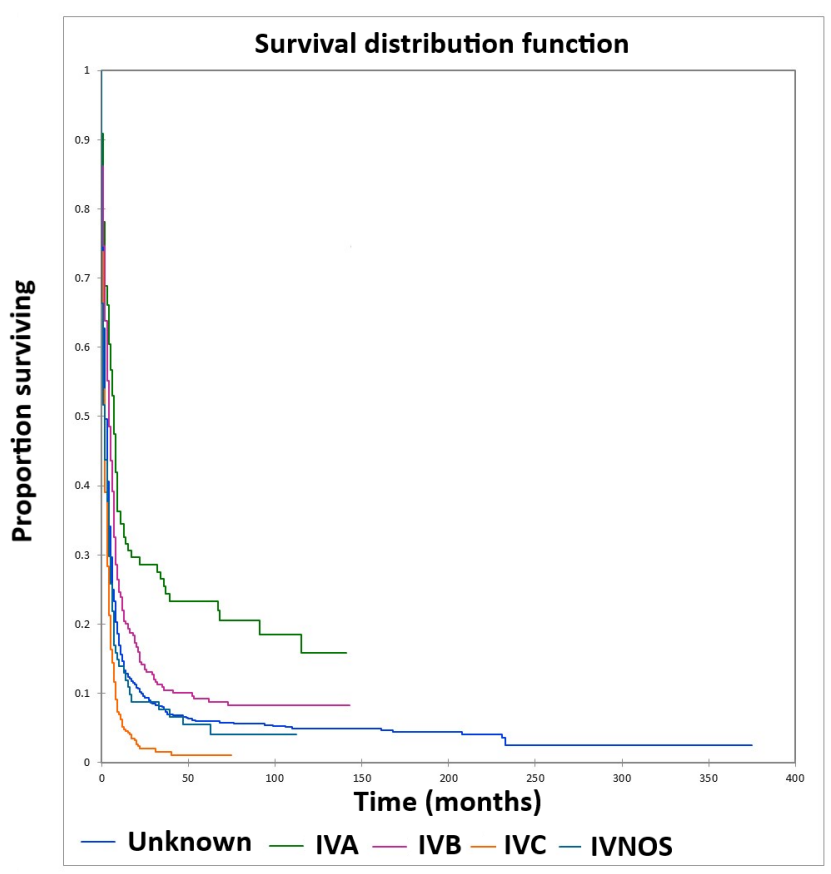

Fig. 9. Kaplan-Meier overall survival by AJCC stage.

TABLE I: PATIENT DEMOGRAPHICS

\begin{tabular}{ccc}
\hline \hline Demographics & $\mathrm{n}$ & $\%$ \\
\hline Total & 1642 & 100 \\
Female & 1021 & 62.2 \\
Male & 621 & 37.8 \\
Black & 124 & 7.6 \\
Other (American Indian/ AK Native, & 191 & 11.6 \\
Asian/ Pacific Islander) & 5 & 0.3 \\
Unknown & 1322 & 80.5 \\
white & 1 & 0.06 \\
15-19 years & 2 & 0.1 \\
25-29 years & 7 & 0.4 \\
30-34 years & 11 & 0.7 \\
35-39 years & 21 & 1.3 \\
40-44 years & 48 & 2.9 \\
45-49 years & 91 & 5.5 \\
50-54 years & 145 & 8.8 \\
55-59 years & 210 & 12.8 \\
60-64 years & 200 & 12.2 \\
65-69 years & 227 & 13.8 \\
70-74 years & 243 & 14.8 \\
$75-79$ years & 209 & 12.7 \\
80-84 years & 227 & 13.8 \\
85+ years & & \\
\hline
\end{tabular}

\begin{tabular}{ccc} 
TABLE II: PATIENT STAGING & \\
\hline \hline AJCC Stage & $\mathrm{N}$ & $\%$ \\
\hline Unknown & 602 & 36.7 \\
IVA & 110 & 6.7 \\
IVB & 368 & 22.4 \\
IVC & 458 & 27.9 \\
IVNOS & 104 & 6.3 \\
T Stage & & \\
Unknown & 602 & 36.7 \\
T4NOS & 121 & 7.4 \\
T4a & 177 & 10.8 \\
T4b & 742 & 45.2 \\
N Stage & & \\
Unknown & 602 & 36.7 \\
N0 & 377 & 23.0 \\
N1NOS & 89 & 5.4 \\
N1a & 102 & 6.2 \\
N1b & 325 & 19.8 \\
NX & 147 & 9.0 \\
M Stage & & \\
Unknown & 602 & 36.7 \\
M0 & 499 & 30.4 \\
M1 & 458 & 27.9 \\
\hline \hline
\end{tabular}

\begin{tabular}{|c|c|c|c|}
\hline \multicolumn{2}{|c|}{$\mathrm{MX}$} & 83 & 5.1 \\
\hline \multicolumn{4}{|c|}{ TABLE III: MULTIVARIATE ANALYSIS } \\
\hline Variable & p-Value & $\begin{array}{c}\text { Hazard } \\
\text { Ratio (HR) }\end{array}$ & $\begin{array}{c}95 \% \text { Confidence } \\
\text { Interval of Hazard } \\
\text { Ratio (HR) } \\
\end{array}$ \\
\hline $\begin{array}{c}\text { Age of } \\
\text { Diagnosis }\end{array}$ & $<0.0001$ & 40.7 & $12.2-125.6$ \\
\hline AJCC Stage & $<0.0001$ & 46.4 & $14.7-146.4$ \\
\hline T Stage & $<0.0001$ & 62.5 & $17.8-250.0$ \\
\hline N Stage & $<0.0001$ & 10.3 & 1.7-89.9 \\
\hline M Stage & $<0.0001$ & 12.5 & $2.5-38.1$ \\
\hline $\begin{array}{l}\text { Surgical vs. } \\
\text { Nonsurgical } \\
\text { Treatment }\end{array}$ & $<0.0001$ & 0.6 & $0.5-0.7$ \\
\hline Race & 0.948 & 1.03 & $0.9-1.1$ \\
\hline Sex & 0.453 & 1.09 & $0.9-1.2$ \\
\hline
\end{tabular}

\section{DISCUSSION}

Anaplastic thyroid carcinoma is the most aggressive histological subtype of thyroid cancer, and the prognosis of patients with ATC is dismal. In the present population-based study including 1642 patients 5-year overall survival was only $6.6 \%$. Previous studies have demonstrated median survival less than 12 months from initial diagnosis [3]-[7]. For patients with surgically resectable disease surgery followed by radiation $+/$ - chemotherapy is often the preferred treatment, but the rarity of this aggressive cancer and the often-advanced stage at diagnosis has made establishment of a standardized treatment regimen difficult. Several studies have suggested that treatment with multimodality therapy, particularly surgery + radiation, may improve survival outcomes in patients with ATC vs. single modality treatment [8]-[10]. The present study analyzed overall survival outcomes in 1642 patients with ATC from the 1973-2015 SEER database. Interestingly, in contrast to [2] race was not found to significantly affect survival in the present study. Reference [2] studied differences in delivery of the recommended treatment and disparities in treatment with multi-modality therapy in black patients vs. white patients appeared to account for the decreased survival outcomes in non-white patients with ATC. In the present study multivariate analysis demonstrated no significant difference in treatment type between black, white, and other patients with ATC, and no significant difference with univariate or multivariate analysis for overall survival with respect to patient race. The difference vs. the Roche et al study is likely a combination of the lack of difference in treatment type, at least with respect to surgical vs. nonsurgical treatment between different racial groups, and possibly also due to the inclusion of the 1973-2015 data in the present study, vs. the 1998-2011 cohort in the Roche study. Reference [11] performed a systematic review of 40 publications on patients with ATC. They noted a median survival of 6.6 months for patients undergoing primary surgery, while the median survival for patients treated with non-surgical therapy was only 2.1 months. They concluded that, when possible, surgery may offer a survival advantage, particularly for patients with stage IVA and stage IVB disease. Reference [12] studied the 2014 SEER database and noted that the incidence of ATC appeared to be increasing over time. They also noted a dismal 
disease specific survival of approximately 4 months. The present study updates that study with the newly available 2015 SEER data on ATC. Reference [13] studied 677 patients from 38 institutions in Japan with ATC with data collected between 1995 and 2008. They noted that distant disease contributed to worse outcomes. They noted 6-month and 1year cause-specific survival rates less than $40 \%$ and $20 \%$, respectively. They noted that age over sev91enty, elevated white blood cell count, T4b tumors, and M1 stage significantly decreased survival. They also noted that in stage IVB patients' aggressive surgery with radiation and chemotherapy appeared to improve cause-specific survival. Reference [14] examined 335 ATC patients from the National Cancer Database from 2003-2006. They noted that in stage IVA patients' positive margins decreased survival, but this survival effect was not seen in IVB and IVC ATC patients. They also noted that on multivariate analysis increased delay in time from diagnosis of ATC to time of surgery did not appear to be associated with decreased survival. Reference [15] and [16] performed a case-control study of 126 ATC patients and 252 controls and a retrospective study on 150 ATC single-institution patients (1995-2005), respectively. Reference [15] found that univariate risk factors for survival in ATC were noted to be lower education, blood type B, goiter, other malignancies, diabetes mellitus, late onset of menarche, and earlier pregnancy, while significant multivariate factors included lower education, blood type B, and goiter. Reference [16] also noted that multivariate survival factors for ATC included increased age, thyroid goiter, surgical treatment, presence of distant metastasis and multifocal tumor. They noted that adjuvant radiation therapy appeared to improve survival in ATC patients. In the present study the mean survival time for patients for whom surgery was not recommended was 7.3 months, while patients who had primary surgery performed had a mean survival time of 30.0 months, reinforcing the likely advantage of surgical treatment in patients amenable to surgery. In the present study age $<45$ years, surgical vs. nonsurgical treatment, and lower overall AJCC, T, N, and M stage were all associated with improved overall survival. Overall survival remained extremely low for all treatment groups. Limitations of the present study include the retrospective nature of the data, which makes recall and selection bias a possibility.

\section{CONCLUSION}

The present study utilized the most recent 1973-2015 SEER database to examine factors influencing overall survival in patients with ATC. Overall survival was low, with $5-, 10-$, and 20 -year survival of $6.6 \%, 5.2 \%$, and $2.7 \%$. Race did not appear to significantly affect overall survival, but lower age, AJCC stage, and T, N, and M stage along with surgical treatment significantly improved survival. Future prospective, multicenter trials might shed light on a standardized treatment regimen for ATC. Surgical treatment with postoperative radiation may improve survival in selected cases with less advanced disease.

\section{ABBREVIATIONS}

AJCC: American Joint Committee on Cancer

DFS: Disease-free survival

M: Metastasis

$\mathrm{N}$ : Nodal

NA: Not available

OS: Overall survival

SEER: Surveillance, Epidemiology, and End Results

T: Tumor

\section{REFERENCES}

[1] Xia Q, Wang W, Xu J, Chen X, Zhong Z, Sun C. Evidence from an updated meta-analysis of the prognostic impacts of postoperative radiotherapy and chemotherapy in patients with anaplastic thyroid carcinoma. OncoTargets and Therapy. 2018; 11: 2251-2257.

[2] Roche AM, Fedewa SA, Shi LL, Chen AY. Treatment and survival vary by race/ethnicity in patients with anaplastic thyroid cancer. Cancer. 2018; 124(8): 1780-1790.

[3] Jereb B, Stjernsward J, Lowhagen T. Anaplastic giant-cell carcinoma of the thyroid. A study of treatment and prognosis. Cancer. 1975; 35(5): 1293-1295.

[4] Spires JR, Schwartz MR, Miller RH. Anaplastic thyroid carcinoma. Association with differentiated thyroid cancer. Archives of Otolaryngology Head and Neck Surgery. 1988; 114(1): 40-44.

[5] Lo CY, Lam KY, Wan KY. Anaplastic carcinoma of the thyroid. American Journal of Surgery. 1999; 177: 337-339.

[6] Nilsson O, Lindeberg J, Zedenius J, Ekman E, Tennvall J, Blomgren $\mathrm{H}$, et al. Anaplastic giant cell carcinoma of the thyroid gland: treatment and survival over a 25-year period. World Journal of Surgery. 1998; 22(7): 725-730.

[7] Tan RK, Finley III RK, Driscoll D, Bakamjian V, Hicks WL, et al. Anaplastic carcinoma of the thyroid: a 24-year experience. Head and Neck. 1995; 17(1): 41-48.

[8] Chen J, Tward JD, Shrieve DC, Hitchcock YJ. Surgery and radiotherapy improves survival in patients with anaplastic thyroid carcinoma: analysis of the surveillance, epidemiology, and end results 1983-2002. American Journal of Clinical Oncology. 2008; 31(5): 460464.

[9] Yau T, Lo CY, Epstein RJ, Lam AK, Wan KY, Lang BH. Treatment outcomes in anaplastic thyroid carcinoma: survival improvement in young patients with localized disease treated by combination of surgery and radiotherapy. Annals of Surgical Oncology. 2008; 15(9): 25002505.

[10] Pierie JP, Muzikansky A, Gaz RD, Faquin WC, Ott MJ. The effect of surgery and radiotherapy on outcome of anaplastic thyroid carcinoma. Annals of Surgical Oncology. 2002; 9(1): 57-64.

[11] Hu S, Helman SN, Hanly E, Likhterov I. The role of surgery in anaplastic thyroid cancer: A systematic review. American Journal of Otolaryngology. 2017; 38(3): 337-350.

[12] Janz TA, Neskey DM, Nguyen SA, Lentsch EJ. Is the incidence of anaplastic thyroid cancer increasing: A population based epidemiology study? The World Journal of Otorhinolaryngology - Head \& Neck Surgery. 2018; 5(1): 34-40.

[13] Sugitani I, Miyauchi A, Sugino K, Okamoto T, Yoshida A, Suzuki S. Prognostic factors and treatment outcomes for anaplastic thyroid carcinoma: ATC Research Consortium of Japan cohort study of 677 patients. World Journal of Surgery. 2012; 36(6): 1247-54.

[14] Goffredo P, Thomas SM, Adam MA, Sosa JA, Roman SA. Impact of timeliness of resection and thyroidectomy margin status on survival for patients with anaplastic thyroid cancer: an analysis of 335 cases. Annals of Surgical Oncology. 2015; 22(13): 4166-74.

[15] Zivaljevic V, Slijepcevic N, Paunovic I, Diklic A, Kalezic N, Marinkovic J, et al. Risk factors for anaplastic thyroid cancer. International Journal of Endocrinology. 2014; 2014: 815070.

[16] Paunovic IR, Sipetic SB, Zoric GV, Diklic AD, Savic DV, Marinkovic $\mathrm{J}$, et al. Survival and prognostic factors of anaplastic thyroid carcinoma. Acta Chirurgica Belgica. 2015; 115: 62-67. 